\title{
Prevalence, severity, and risk factors of disability among adults living with HIV accessing routine outpatient HIV care in London, United Kingdom (UK): A cross-sectional self-report study
}

Darren A Brown ${ }^{1 \pi^{*}}$, Kelly K O’Brien ${ }^{2,3,4 \&}$, Richard Harding ${ }^{5,6 \&}$, Philip Sedgwick ${ }^{7 \pi}$, Mark Nelson $^{8,9 \pi}$, Marta Boffito ${ }^{8 \pi}$, Agnieszka Lewko ${ }^{10 \pi}$

${ }^{1}$ Therapies Department, Chelsea and Westminster Hospital NHS Foundation Trust, London, United Kingdom

${ }^{2}$ Department of Physical Therapy, University of Toronto, Toronto, Canada

${ }^{3}$ Institute of Health Policy, Management and Evaluation (IHPME), University of

Toronto, Toronto, Canada

${ }^{4}$ Rehabilitation Sciences Institute (RSI), University of Toronto, Toronto, Canada

${ }^{5}$ Florence Nightingale Faculty of Nursing, Midwifery \& Palliative Care, King's College London, London, United Kingdom

${ }^{6}$ Cicely Saunders Institute of Palliative Care, Policy and Rehabilitating, King's College London, London, United Kingdom

${ }^{7}$ Institute of Medical and Biomedical Education, St George's University of London, London, United Kingdom

${ }^{8}$ Department of HIV Medicine, Chelsea and Westminster Hospital NHS Foundation Trust, London, United Kingdom

${ }^{9}$ Faculty of Medicine, Department of Medicine, Imperial College London, London, United Kingdom

${ }^{10}$ Centre for Allied Health, Kingston University and St George's University of London, London, United Kingdom

*Corresponding Author

E-mail: Darren.Brown11@nhs.net

I These authors contributed equally to this work

$\&$ These authors also contributed equally to this work

Key Words:

HIV, Disability, Prevalence, Functioning, Physiotherapy, Rehabilitation

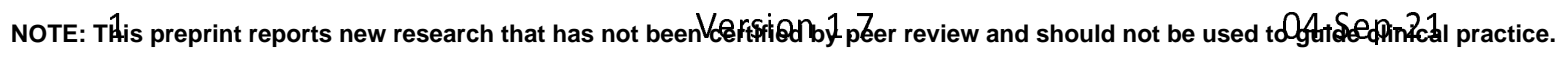




\section{Abstract \\ Objectives}

Measure the prevalence and severity of disability, and examine disability risk factors among adults living with HIV in London, United Kingdom (UK).

\section{Methods}

We conducted a quantitative cross-sectional study. The following self-reported questionnaires were administered: World Health Organization Disability Assessment Schedule 2.0 (WHODAS), HIV Disability Questionnaire (HDQ), a disability item using the Equality Act disability definition (EADD), and demographic and HIV questionnaire. We calculated the proportion (95\% Confidence Interval; $\mathrm{Cl}$ ) of "severe" and "moderate" disability as measured using EADD and WHODAS scores $\geq 2$ respectively. We measured disability severity with $\mathrm{HDQ}$ domain severity scores. We used demographic and HIV characteristic questionnaire responses to assess potential risk factors of "severe" (EADD) and "moderate" (WHODAS scores $\geq 2$ ) disability using logistic regression analysis, and HDQ severity domain scores using linear regression analysis.

\section{Results}

Of 201 participants, 176 (87.6\%) identified as men, with median age 47 years, and 194 (96.5\%) virologically suppressed. Severe disability prevalence was 39.5\% ( $n=79 / 201), 95 \% \mathrm{Cl}$ [32.5\%, 46.4\%]. Moderate disability prevalence was $70.5 \%$ ( $n=141 / 200), 95 \% \mathrm{Cl}[64.2 \%, 76.8 \%]$. Uncertainty was the most severe HDQ disability domain. The HIV characteristic of late HIV diagnosis was a risk factor for severe disability [Odds Ratio (OR) 2.71 ; Cl 1.25, 5.87]. The social determinants of health economic inactivity [OR 2.79; $\mathrm{Cl} 1.08,7.21]$ and receiving benefits [OR 2.87; $\mathrm{Cl} 1.05$, 7.83] were risk factors for "severe" disability. Economic inactivity [OR 3.14; $\mathrm{Cl} 1.00$, 9.98] was a risk factor for "moderate" disability. Economic inactivity, receiving benefits, and having no fixed abode were risk factors $(P \leq 0.05)$ across HDQ disability domains; physical, mental and emotional, difficulty with day-to-day activities, and 
medRxiv preprint doi: https://doi.org/10.1101/2021.09.14.21263587; this version posted September 17, 2021. The copyright holder for this preprint (which was not certified by peer review) is the author/funder, who has granted medRxiv a license to display the preprint in perpetuity.

It is made available under a CC-BY-ND 4.0 International license.

challenges to social participation. Personal factors of identifying as a woman and being aged $<50$ years were risk factors $(P \leq 0.05)$ for HDQ disability domains; mental and emotional, uncertainty, and challenges with social participation.

\section{Conclusions}

People living with well-controlled HIV in London UK experienced multi-dimensional and episodic disability. Results help to better understand the prevalence, severity, and risk factors of disability experienced by adults living with HIV, identify areas to target interventions, reduce disability, and optimise health and function. 


\section{Background}

HIV is now considered a chronic [1] and episodic [2,3] health condition. For the 37.9 million people living with HIV globally [4], universal access to antiretroviral therapies offer normal life expectancy [5]. With access to effective and tolerable antiretroviral therapies, the number of people living with HIV aged 50 years or older are increasing at exponential and unprecedented rates [6]. It is estimated that globally, 7.5 million people living with HIV are aged 50 years or older [7]. Furthermore, by 2028 over half of people living with HIV In the United Kingdom (UK) will be aged $\geq 50$ years [8], with similar patterns observed elsewhere in Europe and North America [9]. As people live longer with chronic HIV, they are susceptible to health conditions arising from the underlying infection, potential side effects of treatments, and ageing [10], resulting in increasingly more prevalent multi-morbidity $[1,11]$. Common concurrent health conditions include cardiovascular disease [12], diabetes [13], bone and joint disorders [14,15], neurocognitive disorders [16,17], chronic pain [18], mental health conditions [19], cancer [20], and frailty [21]. People living with HIV can also experience additional challenges of stigma, ageism, income insecurity, and lack of social support, which may impact or intersect with issues of living and ageing with HIV [22-25]. Collectively the physical, mental and social health challenges experienced can be conceptualised as disability [2]. Many people living and ageing with HIV on long-term antiretroviral therapy now face new or worsening experiences of a wide variety of disability $[10,26]$, with gender, physical symptoms, depression, antiretroviral therapy adherence, and duration living with HIV associated with experiencing disability [27-29].

The conceptualisation of disability is complex and has evolved over time [30]. The predominating framework of disability, the "International Classification of Functioning, Disability and Health" (ICF) [31], combines elements of both medical [32] and social models [33] of disability, leading to a "bio-psycho-social" framework. The ICF describes disability as multidimensional and the outcome of interactions between a person's health condition(s) and context (environmental and personal 
factors), involving one or more dysfunctions at the level of impairments, activity limitations and participation restrictions. The ICF is not specific to any health condition and may not accurately capture the complexity of HIV [2]. The "Episodic Disability Framework" (EDF) presents a new way to conceptualise disability based on the experience of people living with HIV [2]. It conceptualises disability as multidimensional and episodic, describing the health-related consequences of HIV, adverse effects of treatments, and concurrent health conditions that may fluctuate over time [3]. Neither conceptualisation is inherently better than the other. Disability can however be broadly defined as any physical, cognitive, mental or emotional impairments, difficulty with day-to-day activities, challenges to social inclusion, or uncertainty, that can be episodic in nature, whereby disability reflects the interaction between a person's body and the society in which they live [2,34].

Measuring disability is a critical component of care as people live longer with HIV [35] to determine the prevalence and impact of disability [36], prevent discrimination, monitor inequalities, identify service needs and address barriers to equal participation in society [37]. Measuring disability prevalence poses several challenges, not least since there is a wide range of definitions, measurement tools, and purposes for data collection [38]. Measuring impairments alone is not an adequate proxy for disability, since people with the same impairment can experience different types and degrees of restriction [39]. The majority of studies estimating disability prevalence among people living with HIV have focused only on measurements of single impairments [40]. Tools have been developed to standardise disability measurement reflecting the multi-dimensional nature of disability, including generic tools such as the Washington Group on Disability Statistics [41], Equality Act disability definition (EADD) of Great Britain [42], World Health Organization Disability Assessment Schedule 2.0 (WHODAS) [43], and Model Disability Survey [44], alongside condition specific tools such as the HIV Disability Questionnaire (HDQ) [45]. In the UK general population, disability prevalence is estimated with EADD $[42,47]$ to meet the needs of government policy and equalities monitoring [37]. There is no known literature reporting disability prevalence among 
people living with HIV in the UK, using this generic approach to disability measurement within populations.

With the variety of measurement approaches available, each with benefits and limitations, a single measure to estimate disability prevalence might be problematic [38]. It has been proposed that at least two disability prevalence rates should be reported, to represent a range of disability including a moderate threshold and a more severe threshold [38]. To our knowledge there is no known evidence estimating disability prevalence representing a range of disability severity, nor an understanding of potential risk factors for disability among people living with HIV in the UK.

The objectives of our study were to estimate the prevalence and severity of disability, and examine the potential risk factors of disability among adults living with HIV accessing routine in-person outpatient HIV care in London, UK.

\section{Methods}

We conducted a quantitative cross-sectional study, using self-report questionnaires, to measure prevalence and severity of disability and examine potential risk factors of disability, among adults living with HIV accessing routine in-person outpatient HIV care in London, UK. Quality criteria for reporting cross-sectional studies set out in the STROBE statement were used to guide our methodological approach [49].

\section{Patient and public involvement}

Patients and the public were involved throughout the research study design and management. Sources of Patient and Public Involvement (PPI) included host organisation "patient champion", host organisation directorate peer review, and Positively UK; a UK based charity for people living with HIV. 


\section{Ethics}

We obtained ethical approval from NHS Research Ethics Committee and Health Research Authority (REC reference 18/LO/0590; IRAS 236835). All participants provided written informed consent to participate in the study.

\section{Participants}

We recruited adults $\geq 18$ years living with HIV and on antiretroviral HIV treatment for $\geq 6$ months attending routine in-person outpatient HIV care from three outpatient HIV clinics in central London, UK, between May-July 2018. We used convenience sampling, approaching alternative patients in clinic requesting participation in the study.

\section{Sample size}

No prerequisite data exists on prevalence of disability among the estimated 105,200 people living with HIV in the UK [50]. An exploratory sample size of 200 participants was proposed.

\section{Data collection}

Our primary outcome was disability. We measured disability using three approaches, by administering the following three self-completed paper-based questionnaires after routine in-person outpatient HIV care at the clinic visit; (a) demographic and HIV questionnaire including EADD, (b) WHODAS, (c) HDQ.

\section{Measuring disability}

1) EADD: The EADD [42] was developed through a programme of technical development and public consultation following a cross-government Equality Data review in 2007. The EADD tries to reflect the definitions that appeared in legal terms in the Disability Discrimination Act 1995 and the subsequent Equality Act 2010. According to the Equality Act a person is disabled if they have a physical or mental impairment and the impairment has a substantial 
and long-term adverse effect on their ability to carry out normal day-to-day activities [37]. The classification questions making up the EADD are: (a) "do you have any physical or mental health conditions or illnesses lasting or expecting to last 12-months or more?"; (b) "do any of your conditions of illnesses reduce your ability to carry out day-to-day activities?" [42]. A person is counted as disabled if they answer "yes" to both classification questions.

2) WHODAS 2.0 (12 Item): The WHODAS [43] is a self-administered generic measurement tool of functioning and disability applicable across cultures in adult populations, and directly linked to the ICF $[43,51]$. It measures an individual's difficulty in performing specific functions over the previous 30 days across six disability domains: i) cognition, ii) mobility, iii) self-care, iv) getting along, v) life activities and vi) participation). When completing the WHODAS individuals provide an answer for each question on a 5-point Likert scale (range 0-4) with higher scores indicating increasing difficulty completing the task. The WHODAS provides "simple" and "complex" sum scores. In "simple" scoring, the scores assigned to each item are summed (range 0-48) with higher scores suggestive of greater disability [51]. In "complex" or item response theory-based scoring, multiple levels of difficulty are factored for each item using downloadable scoring sheet from the WHODAS website, providing a disability range from 0 (no disability) to 100 (total disability) [51]. The WHODAS has been used among adults living with HIV in high $[29,48,52,53]$, middle-, and low-income countries [28,29,54-58]. The WHODAS has high internal consistency and test-retest reliability $[59,60]$, plus rigorous validity and cross-cultural testing spanning 19 countries [51]. The WHODAS is also validated in patients with chronic diseases [61] and people living with HIV [57]. The short-form WHODAS (12-item) used in our study explains $81 \%$ variance of long-form (36-item), and average short-form administration time is 5 minutes $[43,51]$. For the purpose of disability statistics, categorisation thresholds have been developed based on WHODAS scores, to identify people living with HIV experiencing disability (score $\geq 2$, representing at least two mild/moderate or one moderate/severe limitation) 
$[27,28]$, and any level of functional limitation (score $\geq 1$, representing at least one mild/moderate limitation) $[29,48]$. These thresholds permit prevalence estimates of disability and functional limitation among people living with HIV in South Africa [27-29] and United States [29,48].

3) HDQ: The HDQ is a 69-item self-administered questionnaire developed from the EDF, through a community-academic partnership, to describe the presence, severity and episodic nature of disability experienced by people living with HIV $[2,45]$. The HDQ includes six disability domains: i) physical, ii) cognitive and, iii) mental and emotional health symptoms and impairments, iv) uncertainty, v) difficulty with day-to-day activities, and vi) challenges to social inclusion, and one 'good day/bad day' health classification item. Participants are asked to rate the level of presence and severity of each health challenge on a given day ranging from 0 (not at all) to 4 (extreme). HDQ presence, severity and episodic scores are linearly transformed to a score ranging from 0 to 100, with higher scores indicating a greater presence, severity and episodic nature of disability [62]. The HDQ has demonstrated sensibility, validity, internal consistency reliability, test-retest reliability, and varied precision of measurement in samples of adults living with HIV in Canada, Ireland, United States, and UK [35,53,62-64]. Median administration time is $8-15$ minutes [53].

\section{Demographic and HIV characteristics}

We administered the paper-based self-report demographic and HIV questionnaire which included 19 items capturing: age (years), gender identity, sexual orientation, ethnicity, number of years since HIV diagnosis, whether HIV was diagnosed "late" (CD4 $<350$ cell $/ \mathrm{mm}^{3}$ at diagnosis), antiretroviral therapy use, most recent viral load (copies/ml), employment status, housing situation, use of adaptations to support day-to-day activities, educational attainment, transport, diagnosed concurrent health conditions, health status, receiving or providing care, receipt of benefits, receiving rehabilitation in past 12 -months, and $\operatorname{EADD}[42,47]$. Clinical characteristics 
were collected from electronic records only when participants could not provide data including number of years since HIV diagnosis, most recent viral load, and diagnosed concurrent health conditions.

\section{Data analysis}

\section{Participant demographic, HIV, and disability characteristics}

For continuous variables, we calculated median, Lower Quartile (LQ) and Upper Quartile (UQ), and range including: age, number of years since HIV diagnosis, number of concurrent health conditions, number of transportation modes, and number of rehabilitation professionals received care from in the past 12-months.

For categorical variables, we reported frequencies and percentages including: gender identity, sexual orientation, ethnicity, whether HIV was diagnosed "late" (CD4 $<350$ cell $/ \mathrm{mm}^{3}$ at diagnosis), antiretroviral therapy use, viral load $<50$ copies $/ \mathrm{ml}$, employment status, housing situation, use of adaptations to support day-to-day activities, educational attainment, transport, health status, receiving or providing care, receipt of benefits, and received rehabilitation in past 12-months.

We reported disability characteristics as measured by domain scores of the WHODAS and HDQ. We reported frequency and percentages for WHODAS simple scores in response to all 12-items, presence of any functional limitation (score $\geq 1$ ) per WHODAS domain, WHODAS total number of limitations, and WHODAS difficulty levels. WHODAS "simple" and "complex" sum scores were both reported to follow recommendations to report "complex" sum score [43], and take account of current debate promoting "simple" sum score [65]. Both "simple" and "complex" sum scores were presented as mean and standard deviation (SD), plus median and $25-75^{\text {th }}$ percentile, to align with normative data presented as mean and SD [59] and existing WHODAS 12-item literature reporting either mean and SD, or median and LQ-UQ [66]. We reported median, LQ, UQ, and range for HDQ presence, severity, and episodic total scores and scores per HDQ domain. 


\section{Disability prevalence}

The World Bank recommends estimating disability prevalence with at least two rates representing a range of severity [38]. For the purpose of this study we reported two disability prevalence rates representing "severe" and "moderate" thresholds:

1) Severe disability: We defined severe disability with the EADD, which is used for UK disability national surveillance [67]. This census-based approach may correspond to people with most severe disability [38]. Participants were defined as experiencing "severe" disability if they self-rated "yes" to both EADD classification questions.

2) Moderate disability: We defined moderate disability with the WHODAS, the only known approach estimating disability prevalence among people living with HIV [27-29,48]. This survey-based approach may correspond to people with moderate disability [27]. Participants were defined as experiencing "moderate" disability with WHODAS scores $\geq 2$, representing at least two $\mathrm{mild} /$ moderate or one moderate/severe limitation on WHODAS items $[27,28]$.

We reported disability prevalence representing both severe and moderate thresholds, as frequency, percentage, and 95\% confidence interval (CI).

\section{Disability severity}

We calculated median, LQ and UQ of each of the six HDQ domain severity scores. Interpretability of HDQ scores among people living with HIV remains unknown hence we describe severity on the continuous scale (0-100) whereby higher scores indicated greater severity of disability [35].

\section{Potential risk factors of disability}

We examined potential risk factors of disability using logistic and linear regression analysis for categorical and continuous variables respectively. Our outcome variable 
(disability) was defined using three approaches: (a) severe disability (EADD); (b) moderate disability (WHODAS score $\geq 2$ ); (c) all six HDQ domain severity scores.

The following variables were considered as potential risk factors in the models:

- Age: " $<50$ years "and " $\geq 50$ years", because $\geq 50$ years is the most used definition of "older" within current HIV literature [68,69], and British HIV Association standards of care [70].

- Gender: Where gender breakdowns were presented, Transwomen and Transmen were included in the gender groups with which they self-identified in accordance with Public Health England data on people living with HIV [71], resulting in categorised "man" and "woman".

- Sexual orientation: "Heterosexual" and "Lesbian/Gay/Bisexual/Other" in accordance with UK Government Statistical Service (GSS) harmonised principles [72].

- Ethnicity: "White" and "Black, Asian, and Minority Ethnicities (BAME)" in accordance with UK GSS harmonised principles [73].

- Late HIV diagnosis: Whether HIV was diagnosed "late" was defined by CD4 count $<350$ cell $/ \mathrm{mm}^{3}$ at diagnosis, resulting in categorised "CD4 count $<350$ cell $/ \mathrm{mm}^{3}$ at diagnosis" and "CD4 count $>350$ cell $/ \mathrm{mm}^{3}$ at diagnosis".

- Employment: "economically active" and "economically inactive", based on the Labour Force Survey whereby economic inactivity is defined by people not in employment who have not been seeking work within the last 4 weeks and/or are unable to start work within the next 2 weeks [74].

- Housing situation: "owner occupied/privately rented/social rented" and "no fixed abode/other", based on UK GSS harmonised principles [75].

- Educational attainment: categorised by achieving educational qualifications at "degree level or above" or "any other kind of qualification" according to UK GSS harmonised principles [76].

- Care and Support: "does not receive or provide care" and those who "receive or provide care", whereby receiving care includes social services care and informal/unpaid care [77]. 
- Benefits: "not in receipt of benefits" and "in receipt of benefits" whereby being in receipt of benefits includes receiving working age benefits, disability benefits, pensioner benefits, child benefits, social fund, and/or other benefits, in accordance with UK GSS harmonised principles [78].

- Rehabilitation: "received rehabilitation in past 12-months" and "no rehabilitation in past 12-months" whereby rehabilitation is defined as receiving Physiotherapy, Occupational Therapy, Speech and Language Therapy, and/or complementary and alternative services [79].

We conducted logistic regression analysis to determine risk factors for severe (EADD) and moderate (WHODAS score $\geq 2$ ) disability. We calculated unadjusted and adjusted odds ratios (OR) for each risk factor. We conducted multivariate regression (generalised liner model) for HDQ domain severity scores. We estimated marginal means, mean difference, $95 \% \mathrm{Cl}$, and $P$-values for each risk factor as a potential predictor of disability.[35]

Level of significance was $\leq 0.05$ (5\%). We did not adjust for multiple hypothesis testing, as adjustment using Bonferroni's correction factor tends to be too conservative and result in an excess of Type II errors [80]. We used a pragmatic approach, with greater emphasis given to statistical significance below 0.01 (1\%). Data analysis was performed using SPSS Version 25 [81].

\section{Results}

A total of 316 potential participants were screened for inclusion, with $n=204$ (64.6\%) consenting to participate, and $n=3$ not meeting eligibility criteria of antiretroviral therapy use for $\geq 6$ months. The final sample was $n=201$, with one person not completing all three questionnaires. Median time to complete the questionnaires was 22 minutes (range 9-54 minutes).

\section{Participant characteristics}

\section{Sample demographic and HIV characteristics}


Participant characteristics are shown in Table 1. The majority identified as men (87.6\%), of gay/lesbian sexual orientation (78.6\%), and white ethnicity (72.7\%). The median age was 47 years, with $41.0 \%$ aged $\geq 50$ years. The sample were mostly living with well controlled HIV with all participants taking antiretroviral therapy with $96.5 \%$ achieving HIV viral suppression. Median number of years since HIV diagnosis was 11 years (25-75 ${ }^{\text {th }}$ percentile: 5-21). Half were diagnosed with HIV late (51.7\%).

Participants reported living with a median of two concurrent health conditions in addition to HIV, with most self-reporting general health status "very good" or "good". Participants were mostly economically active, living in owned or private/social rented accommodation, living alone, and half achieving degree level qualifications. The majority did not receive or provide care, receive benefits/tax credits, or receive rehabilitation in past 12-months.

Table 1 - Participant characteristics $(n=201)$

\begin{tabular}{|l|l|}
\hline Age & $\begin{array}{l}\text { Median (LQ, } \\
\text { UQ) [Range] }\end{array}$ \\
\hline Median Age & $\begin{array}{l}47(37,56)[22- \\
88]\end{array}$ \\
\hline Number of participants aged $\geq 50$ years; number (\%) & $82(41.0)$ \\
\hline Gender Identity & Number (\%) \\
\hline Man / Boy & $176(87.6)$ \\
\hline Women / Girl & $20(10.0)$ \\
\hline Transwomen / Transgirl & $5(2.4)$ \\
\hline Sexual Orientation & Number (\%) \\
\hline Gay / Lesbian & $158(78.6)$ \\
\hline Heterosexual / Straight & $31(15.4)$ \\
\hline Bisexual & $8(4.0)$ \\
\hline Don't know / Prefer not to say / Other & $4(2.0)$ \\
\hline Ethnicity & Number (\%) \\
\hline white - English / Welsh / Scottish / Northern Irish / British & $93(46.3)$ \\
\hline white - Irish & $6(3.0)$ \\
\hline white - any other white background & $47(23.4)$ \\
\hline Mixed / Multiple Ethnic groups - white and Black Caribbean & $4(2.0)$ \\
\hline Mixed / Multiple Ethnic groups - white and Black African & $5(2.5)$ \\
\hline Mixed / Multiple Ethnic groups - white and Asian & $1(0.5)$ \\
\hline Mixed / Multiple Ethnic groups - any other mixed / multiple & $4(2.0)$ \\
\hline Ethnic background & \\
\hline Asian / Asian British - Indian & $6(3.0)$ \\
\hline Asian / Asian British - Pakistani & $1(0.5)$ \\
\hline & \\
\hline
\end{tabular}




\begin{tabular}{|c|c|}
\hline Asian / Asian British - Chinese & $1(0.5)$ \\
\hline Asian / Asian British - any other Asian background & $2(1.0)$ \\
\hline Black / Black British - African & $22(10.9)$ \\
\hline Black / Black British - Caribbean & $4(2.0)$ \\
\hline $\begin{array}{l}\text { Black / Black British - any other Black / African / Caribbean } \\
\text { background }\end{array}$ & $1(0.5)$ \\
\hline Other Ethnic group - Arab & $2(1.0)$ \\
\hline Other Ethnic group - any other Ethnic group & $2(1.0)$ \\
\hline Number of years since HIV diagnosis & $\begin{array}{l}\text { Median (LQ, } \\
\text { UQ) [Range] }\end{array}$ \\
\hline Median years since HIV diagnosis & $11(5,21)[1-37$ \\
\hline HIV diagnosed late & Number (\%) \\
\hline CD4 count $<350$ cell $/ \mathrm{mm}^{3}$ at diagnosis & $104(51.7)$ \\
\hline Taking Antiretroviral Therapy & Number (\%) \\
\hline Yes & $201(100.0)$ \\
\hline Viral Load Undetectable & Number (\%) \\
\hline Viral load $<50$ copies $/ \mathrm{ml}$ & $194(96.5)$ \\
\hline Concurrent health conditions & $\begin{array}{l}\text { Median (LQ, } \\
\text { UQ) [Range] }\end{array}$ \\
\hline Median number of conditions in addition to living with HIV & $2(1,5)[0-18]$ \\
\hline Self-rated general health status & Number (\%) \\
\hline Very good & $78(38.8)$ \\
\hline Good & $74(36.8)$ \\
\hline Fair & $35(17.4)$ \\
\hline Poor & $14(7.0)$ \\
\hline Employment & Number (\%) \\
\hline Self-employed & $42(20.9)$ \\
\hline Full-time employed & $70(39.3)$ \\
\hline Part-time employed & $13(6.5)$ \\
\hline Not working; available to start work in 2 weeks & $4(2.0)$ \\
\hline Not working; looked for work in past 4 weeks & $3(1.5)$ \\
\hline Waiting to start a new job & $2(1.0)$ \\
\hline Unemployed & $14(7.0)$ \\
\hline Retired & $20(10.0)$ \\
\hline Full time student / at school & $4(2.0)$ \\
\hline Long term sick or disabled & $20(10.0)$ \\
\hline Accommodation & Number (\%) \\
\hline Owner occupied & 75 (37.3) \\
\hline Privately rented accommodation & $73(36.3)$ \\
\hline Social rented housing & $46(22.9)$ \\
\hline No fixed abode & $3(1.5)$ \\
\hline Other & $4(2.0)$ \\
\hline Household information & Number (\%) \\
\hline Lives alone & $89(44.3)$ \\
\hline Lives with friends & $32(15.9)$ \\
\hline Lives with family & $36(17.9)$ \\
\hline
\end{tabular}




\begin{tabular}{|c|c|}
\hline Lives with children & $6(3.0)$ \\
\hline Other & $38(18.9)$ \\
\hline Housing adaptations to support day-today activities & Number (\%) \\
\hline No & $171(85.1)$ \\
\hline Educational Attainment & Number (\%) \\
\hline Attained educational qualifications & $185(92.0)$ \\
\hline Attained professional / vocational qualifications & $133(66.2)$ \\
\hline Highest educational qualification & Number (\%) \\
\hline Degree level or above & $117(58.2)$ \\
\hline Any other kind of qualification & $73(36.3)$ \\
\hline No qualifications & $11(5.5)$ \\
\hline Modes of transportation to appointment & Number (\%) \\
\hline Underground (tube) & $83(41.3)$ \\
\hline Bus & $60(29.9)$ \\
\hline Walk & $49(24.4)$ \\
\hline Train & $42(20.9)$ \\
\hline Car & $22(10.9)$ \\
\hline Bike & $7(3.5)$ \\
\hline Other & $9(4.5)$ \\
\hline Number of transport modes & $\begin{array}{l}\text { Median (LQ, } \\
\text { UQ) [Range] }\end{array}$ \\
\hline Median number of transportation modes & $1(1,2)[1-4]$ \\
\hline Care and support & Number (\%) \\
\hline Receive care from social services & $10(5.0)$ \\
\hline Receive informal / unpaid care & $19(9.5)$ \\
\hline Provide care for others (eg: friends or family) & $29(14.4)$ \\
\hline Do not receive of provide care & $145(72.1)$ \\
\hline Benefits and tax credits & Number (\%) \\
\hline Working age benefits & $36(17.9)$ \\
\hline Disability benefits & $36(17.9)$ \\
\hline Child benefits & $8(4.0)$ \\
\hline Pensioner benefits & $10(5.0)$ \\
\hline Social fund & $1(0.5)$ \\
\hline Other benefits & $1(0.5)$ \\
\hline None & $141(70.1)$ \\
\hline $\begin{array}{l}\text { Received care from rehabilitation professionals in past 12- } \\
\text { months }\end{array}$ & Number (\%) \\
\hline Physiotherapy & $51(25.4)$ \\
\hline Occupational Therapy & $15(7.5)$ \\
\hline Speech and Language Therapy & $2(1.0)$ \\
\hline Complimentary and alternative services & $29(14.4)$ \\
\hline None & $130(64.7)$ \\
\hline $\begin{array}{l}\text { Number of rehabilitation professionals accessed in past 12- } \\
\text { months }\end{array}$ & $\begin{array}{l}\text { Median (LQ, } \\
\text { UQ) [Range] }\end{array}$ \\
\hline Median number of rehabilitation professionals & $0(0,1)[0-3]$ \\
\hline
\end{tabular}




\section{Sample disability characteristics}

The WHODAS simple scores in response to all 12-items are shown in Supplemental File Table 1. Frequency of any functional limitation (score $\geq 1$ ) within each of the six WHODAS disability domains were; challenges to social participation $(n=208,52.0 \%)$, challenges getting along ( $n=150,37.5 \%)$, challenges to life activities ( $n=166,41.5 \%)$, cognitive health challenges ( $n=147,36.7 \%)$, mobility challenges $(n=145,36.2 \%)$, and challenges with self-care ( $n=90,22.5 \%)$. Any level of functional limitation (WHODAS score $\geq 1$ ) was reported by $n=159$ (79.5\%), whereby $n=26$ (13.0\%) scored one limitation, $n=18$ (9.0) two limitations, $n=14$ (7.0\%) three limitations, $n=101$ (50.5\%) four or more limitations, and $n=21$ (10.5\%) scoring all twelve limitations. Difficulty levels across all WHODAS items were "mild difficulty" $n=333$ (38.8\%), "moderate difficulty" n=305 (33.7\%), "severe difficulty" n=143 (15.8\%), and "extreme difficulty/cannot do" n=125 (13.8\%). WHODAS "simple" sum score were mean 9.4 (SD; 11.3), median 5.0 (25-75 ${ }^{\text {th }}$ percentile; 1.0-12.0). WHODAS "complex" sum score were mean 19.6 (SD; 23.7), median 10.4 (LQ-UQ; 2.1-25.6). The HDQ total and domain scores are shown in Table 2. The most present, severe and episodic HDQ disability domains were "uncertainty", "uncertainty", and "physical symptoms and impairments" respectively, with $n=177$ (88.5\%) reported completing the HDQ on a good day. 
Table 2: Median HDQ domain scores $(n=200)$

\begin{tabular}{|c|c|c|c|}
\hline HDQ domain (\# items) & $\begin{array}{l}\text { HDQ Presence } \\
\text { Score: } \\
\text { Median (LQ, UQ) } \\
\text { [Range] }\end{array}$ & $\begin{array}{l}\text { HDQ Severity } \\
\text { Score: } \\
\text { Median (LQ, UQ) } \\
\text { [Range] }\end{array}$ & $\begin{array}{l}\text { HDQ Episodic } \\
\text { Score: } \\
\text { Median (LQ, UQ) } \\
\text { [Range] } \\
\end{array}$ \\
\hline $\begin{array}{l}\text { Physical symptoms and impairments } \\
\text { (20 items) }\end{array}$ & $\begin{array}{l}30.0(15.0,53.8) \\
{[0.0-100.0]}\end{array}$ & $\begin{array}{l}11.3(5.0,26.3) \\
{[0.0-83.8]}\end{array}$ & $\begin{array}{l}20.0(10.0,40.0) \\
{[0.0-100.0]}\end{array}$ \\
\hline $\begin{array}{l}\text { Cognitive symptoms and impairments } \\
\text { ( } 3 \text { items) }\end{array}$ & $\begin{array}{l}33.3(0.0,100.0) \\
{[0.0-100.0]}\end{array}$ & $\begin{array}{l}8.3(0.0,25.0) \\
{[0.0-100.0]}\end{array}$ & $\begin{array}{l}0.0(0.0,66.7) \\
{[0.0-100.0]}\end{array}$ \\
\hline $\begin{array}{l}\text { Mental and emotional health symptoms and impairments } \\
\text { (11 items) }\end{array}$ & $\begin{array}{l}45.5(18.2,72.7) \\
{[0.0-100.0]}\end{array}$ & $\begin{array}{l}13.6(4.5,34.1) \\
{[0.0-100.0]} \\
\end{array}$ & $\begin{array}{l}18.2(0.0,54.5) \\
{[0.0-100.0]}\end{array}$ \\
\hline $\begin{array}{l}\text { Uncertainty or worry about the future } \\
\text { (14 items) }\end{array}$ & $\begin{array}{l}57.1(28.6,78.6) \\
{[0.0-100.0]}\end{array}$ & $\begin{array}{l}23.2(10.7,38.9) \\
{[0.0-100.0]}\end{array}$ & $\begin{array}{l}7.1(0.0,42.9) \\
{[0.0-92.9]}\end{array}$ \\
\hline $\begin{array}{l}\text { Difficulties with day-to-day activities } \\
\text { (9 items) }\end{array}$ & $\begin{array}{l}11.1(0.0,44.4) \\
{[0.0-100.0]} \\
\end{array}$ & \begin{tabular}{|l|}
$2.8(0.0,6.7)$ \\
{$[0.0-80.6]$} \\
\end{tabular} & $\begin{array}{l}0.0(0.0,22.2) \\
{[0.0-100.0]} \\
\end{array}$ \\
\hline $\begin{array}{l}\text { Challenges to taking part in social and community life } \\
\text { (12 items) }\end{array}$ & $\begin{array}{l}33.3(8.3,58.3) \\
{[0.0-100.0]} \\
\end{array}$ & $\begin{array}{l}14.6(4.2,29.2) \\
{[0.0-100.0]} \\
\end{array}$ & $\begin{array}{l}0.0(0.0,25.0) \\
{[0.0-83.3]}\end{array}$ \\
\hline Total HDQ Score & $\begin{array}{l}36.2(21.7,59.4) \\
{[0.0-98.6]}\end{array}$ & $\begin{array}{l}13.4(6.3,28.8) \\
{[0.0-86.6]}\end{array}$ & $\begin{array}{l}17.4(5.8,36.2) \\
{[0.0-97.8]}\end{array}$ \\
\hline
\end{tabular}

Higher scores indicate greater presence, severity and episodic nature of disability.

Bold indicates the highest scores across all domains. 


\section{Disability prevalence}

The estimated prevalence of severe disability was $39.5 \%(n=79), 95 \% \mathrm{Cl}[32.5 \%$, $46.4 \%]$. In total $102(50.7 \%)$ respondents reported presence of any physical or mental health conditions or illnesses lasting or expecting to last 12-months or more, whilst 35 (34.3\%) reported subsequent activity restrictions "Yes, a lot", and 44 (43.1\%) "Yes, a little".

The estimated prevalence of moderate disability was 70.5\%, 95\% Cl [64.2\%, 76.8\%], with 141 respondents scoring $\geq 2$ on WHODAS.

\section{Disability severity}

The HDQ domain severity scores are shown in Table 2 . The three highest severity scores were disability domains: uncertainty or worry about the future (median; LQ, UQ: $23.2 ; 10.7,38.9)$; challenges to taking part in social and community life (14.6; 4.2, 29.2); mental and emotional health symptoms and impairments $(13.6 ; 4.5,34.1)$.

\section{Potential risk factors of disability}

\section{Risk factors of severe disability}

Participants who were diagnosed with HIV late (OR 2.71; 95\% Cl: 1.25, 5.87), economically inactive (OR 2.79; $95 \% \mathrm{Cl}: 1.08,7.21$ ), received benefits (OR 2.87; 95\% $\mathrm{Cl}: 1.05,7.83$ ), and received rehabilitation in past 12-months (OR 4.56; $95 \% \mathrm{Cl}: 2.11$, 9.86) were associated with statistically significant increased odds for "severe" disability (Table 3).

\section{Risk factors of moderate disability}

Participants who were economically inactive (OR 3.14; $95 \% \mathrm{Cl}: 1.00,9.89$ ), and received rehabilitation in past $12-$ months (OR 3.41; $95 \% \mathrm{Cl}: 1.44,8.10$ ) were associated with statistically significant increased odds for "moderate" disability 
medRxiv preprint doi: https://doi.org/10.1101/2021.09.14.21263587; this version posted September 17, 2021. The copyright holder for this preprint (which was not certified by peer review) is the author/funder, who has granted medRxiv a license to display the preprint in perpetuity.

It is made available under a CC-BY-ND 4.0 International license.

(Table 3). All participants categorised as no fixed abode met threshold for moderate disability, therefore associations could not be analysed. 
Table 3: Potential risk factors as predictors of severe or moderate disability

\begin{tabular}{|c|c|c|c|c|}
\hline \multirow[t]{2}{*}{ Characteristic } & \multicolumn{2}{|c|}{$\begin{array}{c}\text { Severe disability } \\
\text { (EADD) }\end{array}$} & \multicolumn{2}{|c|}{$\begin{array}{l}\text { Moderate disability } \\
\text { (WHODAS score } \geq 2 \text { ) }\end{array}$} \\
\hline & $\begin{array}{c}\text { Unadjusted Odds } \\
\text { Ratio }(95 \% \mathrm{Cl})\end{array}$ & $\begin{array}{l}\text { Adjusted Odds } \\
\text { Ratio }(95 \% \mathrm{Cl})\end{array}$ & $\begin{array}{l}\text { Unadjusted Odds } \\
\text { Ratio }(95 \% \mathrm{Cl})\end{array}$ & $\begin{array}{c}\text { Adjusted Odds Ratio } \\
(95 \% \mathrm{Cl})\end{array}$ \\
\hline $\begin{array}{l}\text { Age } \\
\quad<50 \text { years } \\
\geq 50 \text { years }\end{array}$ & $\begin{array}{c}(1) \\
3.33(1.84,6.02)\end{array}$ & $\begin{array}{c}(1) \\
1.02(0.43,2.39)\end{array}$ & $\begin{array}{c}(1) \\
1.25(0.67,2.33)\end{array}$ & $\begin{array}{c}(1) \\
0.43(0.18,1.03)\end{array}$ \\
\hline $\begin{array}{l}\text { Gender Identity } \\
\text { Man } \\
\text { Woman }\end{array}$ & $\begin{array}{c}(1) \\
1.50(0.65,3.48)\end{array}$ & $\begin{array}{c}(1) \\
1.72(0.34,8.83)\end{array}$ & $\begin{array}{c}(1) \\
5.56(1.27,24.38)\end{array}$ & $\begin{array}{c}(1) \\
1.55(0.22,10.98)\end{array}$ \\
\hline $\begin{array}{l}\text { Sexual Orientation } \\
\text { Heterosexual } \\
\text { Lesbian/Gay/Bisexual/Other }\end{array}$ & $\begin{array}{c}(1) \\
0.55(0.26,1.19)\end{array}$ & $\begin{array}{c}(1) \\
1.24(0.26,6.01)\end{array}$ & $\begin{array}{c}(1) \\
0.14(0.03,0.59)\end{array}$ & $\begin{array}{c}(1) \\
0.13(0.01,1.43)\end{array}$ \\
\hline $\begin{array}{l}\text { Ethnicity } \\
\text { White } \\
\text { BAME }\end{array}$ & $\begin{array}{c}(1) \\
0.76(0.40,1.44)\end{array}$ & $\begin{array}{c}(1) \\
0.38(0.14,1.03)\end{array}$ & $\begin{array}{c}(1) \\
1.72(0.83,3.56)\end{array}$ & $\begin{array}{c}(1) \\
0.92(0.38,2.25)\end{array}$ \\
\hline $\begin{array}{l}\text { Late HIV Diagnosis } \\
\text { CD4 count } \geq 350 \text { cell } / \mathrm{mm}^{3} \text { at diagnosis } \\
\text { CD4 count }<350 \text { cell } / \mathrm{mm}^{3} \text { at diagnosis }\end{array}$ & $\begin{array}{c}(1) \\
3.75(2.05,6.88)\end{array}$ & $\begin{array}{c}(1) \\
2.71(1.25,5.87)\end{array}$ & $\begin{array}{c}(1) \\
1.91(1.03,3.55)\end{array}$ & $\begin{array}{c}(1) \\
1.31(0.61,2.79)\end{array}$ \\
\hline $\begin{array}{l}\text { Employment } \\
\text { Economically Active } \\
\text { Economic Inactivity }\end{array}$ & $\begin{array}{c}(1) \\
6.56(3.36,12.79)\end{array}$ & $\begin{array}{c}(1) \\
2.79(1.08,7.21)\end{array}$ & $\begin{array}{c}(1) \\
4.47(1.89,10.57)\end{array}$ & $\begin{array}{c}(1) \\
3.14(1.00,9.89)\end{array}$ \\
\hline $\begin{array}{l}\text { Housing Situation } \\
\text { Owner/Private Rent/Social Rent } \\
\text { No fixed abode / other }\end{array}$ & $\begin{array}{c}(1) \\
4.05(0.77,21.43)\end{array}$ & $\begin{array}{c}(1) \\
2.26(0.32,16.04)\end{array}$ & $\begin{array}{c}(1) \\
-\end{array}$ & $\begin{array}{c}(1) \\
-\end{array}$ \\
\hline $\begin{array}{l}\text { Educational Attainment } \\
\text { Degree level or above } \\
\text { Any other kind of qualification }\end{array}$ & $\begin{array}{c}(1) \\
1.36(0.74,2.48)\end{array}$ & $\begin{array}{c}(1) \\
0.89(0.41,1.92)\end{array}$ & $\begin{array}{c}(1) \\
1.81(0.92,3.56)\end{array}$ & $\begin{array}{c}(1) \\
1.62(0.75,3.49)\end{array}$ \\
\hline Care and Support & & & & \\
\hline
\end{tabular}




\begin{tabular}{|c|c|c|c|c|}
\hline $\begin{array}{l}\text { Does not receive or provide care } \\
\text { Receives or provides care }\end{array}$ & $\begin{array}{c}(1) \\
3.43(1.81,6.52)\end{array}$ & $\begin{array}{c}(1) \\
1.19(0.46,3.09)\end{array}$ & $\begin{array}{c}(1) \\
3.96(1.67,9.37)\end{array}$ & $\begin{array}{c}(1) \\
1.79(0.61,5.25)\end{array}$ \\
\hline $\begin{array}{l}\text { Benefits } \\
\text { Not in receipt of benefits } \\
\text { Receives benefits }\end{array}$ & $\begin{array}{c}(1) \\
6.94(3.53,13.67)\end{array}$ & $\begin{array}{c}(1) \\
2.87(1.05,7.83)\end{array}$ & $\begin{array}{c}(1) \\
4.34(1.84,10.26)\end{array}$ & $\begin{array}{c}(1) \\
1.38(0.41,4.67)\end{array}$ \\
\hline $\begin{array}{l}\text { Rehabilitation } \\
\text { No rehabilitation in past } 12 \text { months } \\
\text { Received rehabilitation in past } 12 \text { months }\end{array}$ & $\begin{array}{c}(1) \\
5.63(2.99,10.60)\end{array}$ & $\begin{array}{c}(1) \\
4.56(2.11,9.86)\end{array}$ & $\begin{array}{c}(1) \\
3.63(1.70,7.74)\end{array}$ & $\begin{array}{c}(1) \\
3.41(1.44,8.10)\end{array}$ \\
\hline
\end{tabular}

Bold indicates $95 \% \mathrm{Cl}$ does not cross unity, and therefore OR statistically significantly different from unity $(P \leq 0.05)$. 


\section{Risk Factors of HDQ domains}

Multivariate associations between potential risk factors and HDQ domains are shown in Table 4.

Physical symptoms and impairments mean severity HDQ domain scores were higher among participants who identified as women ( $95 \% \mathrm{Cl}: 1.22,18.41 ; P=0.025)$, were economically inactive (4.51, $15.54 ; P<0.001)$, of no fixed abode $(3.13,24.86 ; P=0.012)$, received benefits $(1.88,13.52 ; P=0.010)$, and received rehabilitation in past 12 -months $(30.20,42.89 ; P<0.001)$.

Cognitive symptoms and impairments mean severity HDQ domain scores were higher among participants who were economically inactive $(4.88,21.91 ; P=0.002)$, received benefits $(3.73,21.72$; $P=0.006)$, and received rehabilitation $(29.45,49.05 ; P=0.007)$ in past 12 -months.

Mental and emotional health symptoms and impairments mean severity HDQ domain scores were higher among participants aged $<50$ years $(-14.09,-1.23 ; P=0.020)$, who identified as women (1.85, 25.72; $P=0.024)$, were economically inactive $(7.76,23.07 ; P<0.001)$, of no fixed abode $(7.37,37.54$; $P=0.004)$, and received benefits $(0.19,16.36 ; P=0.045)$.

Uncertainty mean severity HDQ domain scores were higher among participants aged $<50$ years $(-13.41$, -0.79; $P=0.027)$, who identified as women $(8.52,31.96 ; P=0.001)$, were economically inactive $(0.25$, 15.29; $P=0.043)$, of no fixed abode $(2.26,31.89 ; P=0.024)$, and received rehabilitation the past $12-$ months $(45.82,63.12 ; P=0.004)$.

Difficulties with day-to-day activities mean severity HDQ domain scores were higher among participants who were economically inactive $(9.92,22.01 ; P<0.001)$, of no fixed abode $(0.02,23.86$; $P=0.050)$, received benefits $(4.19,16.97 ; P=0.001)$, and received rehabilitation the past 12 -months $(28.65,42.57 ; P<0.001)$.

Challenges to taking part in social and community life mean severity HDQ domain scores were higher among participants who were aged $<50$ years $(-11.08,-0.13 ; P=0.045)$, identified as women $(4.28$, 24.62; $P=0.005)$, were economically inactive $(6.25,19.30 ; P<0.001)$, of no fixed abode $(3.23,28.95$; $P=0.014)$, received benefits $(1.63,15.41 ; P=0.015)$, and received rehabilitation in past 12 -months $(35.47,50.49 ; P=0.001)$. 
Table 4: Potential risk factors as predictors of HDQ domains

\begin{tabular}{|c|c|c|c|c|c|c|}
\hline Potential Risk Factors & $\begin{array}{l}\text { HDQ Domain "Physical } \\
\text { symptoms and } \\
\text { impairments"; } \\
\text { Estimated marginal } \\
\text { mean of severity score } \\
(95 \% \mathrm{Cl})\end{array}$ & $\begin{array}{l}\text { HDQ Domain } \\
\text { "Cognitive symptoms } \\
\text { and impairments"; } \\
\text { Estimated marginal } \\
\text { mean of severity score } \\
(95 \% \mathrm{Cl})\end{array}$ & $\begin{array}{l}\text { HDQ Domain "Mental } \\
\text { and emotional health } \\
\text { symptoms and } \\
\text { impairments"; } \\
\text { Estimated marginal } \\
\text { mean of severity score } \\
(95 \% \mathrm{Cl})\end{array}$ & $\begin{array}{l}\text { HDQ Domain } \\
\text { "Uncertainty or worry } \\
\text { about the future"; } \\
\text { Estimated marginal } \\
\text { mean of severity score } \\
(95 \% \mathrm{Cl})\end{array}$ & $\begin{array}{l}\text { HDQ Domain } \\
\text { "Difficulties with day- } \\
\text { to-day activities"; } \\
\text { Estimated marginal } \\
\text { mean of severity score } \\
(95 \% \mathrm{Cl})\end{array}$ & $\begin{array}{l}\text { HDQ Domain } \\
\text { "Challenges to taking } \\
\text { part in social and } \\
\text { community life"; } \\
\text { Estimated marginal } \\
\text { mean of severity score } \\
(95 \% \mathrm{Cl})\end{array}$ \\
\hline $\begin{array}{l}\text { Age } \\
<50 \text { years } \\
\geq 50 \text { years } \\
\text { Mean Difference }(95 \% \mathrm{Cl}) \\
P \text {-value }\end{array}$ & $\begin{array}{l}31.26(25.16,37.36) \\
33.61(27.26,39.97) \\
2.35(-2.27,6.98) \\
P=0.319\end{array}$ & $\begin{array}{l}36.62(27.19,46.05) \\
32.93(23.11,42.75) \\
-3.69(-10.84,3.46) \\
P=0.312\end{array}$ & $\begin{array}{l}44.86(36.39,53.34) \\
37.21(28.38,46.03) \\
-7.66(-14.09,-1.23) \\
P=0.020\end{array}$ & $\begin{array}{l}53.87(45.55,62.19) \\
46.76(38.10,55.43) \\
-7.11(-13.41,-0.79) \\
P=0.027\end{array}$ & $\begin{array}{l}30.26(23.57,36.96) \\
31.31(24.33,38.28) \\
1.05(-4.03,6.12) \\
P=0.686\end{array}$ & $\begin{array}{l}41.41(34.19,48.63) \\
35.80(28.28,43.32) \\
-5.61(-11.08,-0.13) \\
P=\mathbf{0 . 0 4 5}\end{array}$ \\
\hline $\begin{array}{l}\text { Gender Identity } \\
\text { Man } \\
\text { Woman } \\
\text { Mean Difference }(95 \% \mathrm{Cl}) \\
P \text {-value }\end{array}$ & $\begin{array}{l}27.53(21.31,33.75) \\
37.34(29.27,45.41) \\
9.81(\mathbf{1 . 2 2}, \mathbf{1 8 . 4 1 )} \\
\boldsymbol{P}=\mathbf{0 . 0 2 5}\end{array}$ & $\begin{array}{l}32.80(23.18,42.41) \\
36.75(24.28,49.23) \\
3.96(-9.32,17.24) \\
P=0.559\end{array}$ & $\begin{array}{l}34.14(25.50,42.78) \\
47.93(36.71,59.14) \\
13.78(1.85,25.72) \\
P=\mathbf{0 . 0 2 4}\end{array}$ & $\begin{array}{l}40.20(31.71,48.68) \\
60.44(49.43,71.44) \\
20.24(8.52,31.96) \\
P=0.001\end{array}$ & $\begin{array}{l}27.34(20.52,34.17) \\
34.23(25.37,43.09) \\
6.89(-2.54,16.32) \\
P=0.152\end{array}$ & $\begin{array}{l}31.38(24.02,38.74) \\
45.83(36.28,55.38) \\
14.45(4.28,24.62) \\
P=0.005\end{array}$ \\
\hline $\begin{array}{l}\text { Sexual Orientation } \\
\text { Heterosexual } \\
\text { Lesbian/Gay/Bisexual/Other } \\
\text { Mean Difference }(95 \% \mathrm{Cl}) \\
P \text {-value }\end{array}$ & $\begin{array}{l}30.96(23.96,37.97) \\
33.91(26.73,41.08) \\
2.95(-5.25,11.14) \\
P=0.481\end{array}$ & $\begin{array}{l}36.74(25.91,47.56) \\
32.81(21.73,43.90) \\
-3.92(-16.59,8.75) \\
P=0.544\end{array}$ & $\begin{array}{l}39.16(29.44,48.89) \\
42.91(32.94,52.87) \\
3.75(-7.64,15.13) \\
P=0.519\end{array}$ & $\begin{array}{l}54.19(44.64,63.74) \\
46.45(36.66,56.23) \\
-7.74(-18.92,3.44) \\
P=0.175\end{array}$ & $\begin{array}{l}32.42(24.74,40.11) \\
29.15(21.28,37.02) \\
-3.28(-12.27,5.72) \\
P=0.475\end{array}$ & $\begin{array}{l}38.07(29.78,46.36) \\
39.14(30.65,47.63) \\
1.07(-8.63,10.77) \\
P=0.829\end{array}$ \\
\hline $\begin{array}{l}\text { Ethnicity } \\
\text { White } \\
\text { BAME } \\
\text { Mean Difference (95\% Cl) } \\
\text { P-value }\end{array}$ & $\begin{array}{l}32.78(25.56,38.99) \\
32.10(25.81,38.38) \\
-0.68(-5.41,4.04) \\
P=0.778\end{array}$ & $\begin{array}{l}37.89(28.29,47.49) \\
31.66(21.94,41.37) \\
-6.24(-13.54,1.06) \\
P=0.094\end{array}$ & $\begin{array}{l}42.01(33.38,50.64) \\
40.06(31.33,48.79) \\
-1.95(-8.51,4.61) \\
P=0.560\end{array}$ & $\begin{array}{l}50.90(42.43,59.37) \\
49.73(41.16,58.30) \\
-1.17(-7.61,5.27) \\
P=0.722\end{array}$ & $\begin{array}{l}32.04(25.23,38.86) \\
29.53(22.63,36.43) \\
-2.51(-7.69,2.67) \\
P=0.343\end{array}$ & $\begin{array}{l}39.80(32.45,47.15) \\
37.41(29.97,44.85) \\
-2.39(-7.98,3.21) \\
P=0.403\end{array}$ \\
\hline $\begin{array}{l}\text { Late HIV Diagnosis } \\
\text { No } \\
\text { Yes } \\
\text { Mean Difference }(95 \% \mathrm{CI}) \\
\text { P-value }\end{array}$ & $\begin{array}{l}31.05(24.74,37.36) \\
33.83(27.88,39.78) \\
2.78(-1.29,6.85) \\
P=0.181\end{array}$ & $\begin{array}{l}32.18(22.43,41.93) \\
37.37(28.18,46.56) \\
5.19(-1.10,11.48) \\
P=0.106\end{array}$ & $\begin{array}{l}38.51(29.74,47.27) \\
43.56(35.30,51.83) \\
5.06(-0.60,10.71) \\
P=0.080\end{array}$ & $\begin{array}{l}48.58(39.98,57.19) \\
52.05(43.94,60.16) \\
3.47(-2.09,9.02) \\
P=0.221\end{array}$ & $\begin{array}{l}28.59(21.67,35.51) \\
32.98(26.45,39.51) \\
4.39(-0.08,8.86) \\
P=0.054\end{array}$ & $\begin{array}{l}37.65(30.18,45.12) \\
39.56(32.52,46.60) \\
1.91(-2.91,6.73) \\
P=0.437\end{array}$ \\
\hline $\begin{array}{l}\text { Employment } \\
\text { Economically active } \\
\text { Economic inactivity } \\
\text { Mean Difference }(95 \% \mathrm{Cl}) \\
P \text {-value }\end{array}$ & $\begin{array}{l}27.42(20.95,33.90) \\
37.45(31.11,43.79) \\
10.02(4.51,15.54) \\
P<0.001\end{array}$ & $\begin{array}{l}28.08(18.07,38.09) \\
41.47(31.68,51.27) \\
13.39(4.88,21.91) \\
P=0.002\end{array}$ & $\begin{array}{l}33.33(24.33,42.32) \\
48.74(39.94,57.55) \\
15.41(7.76,23.07) \\
P<0.001\end{array}$ & $\begin{array}{l}46.43(37.60,55.26) \\
54.20(45.56,62.84) \\
7.77(0.25,15.29) \\
P=\mathbf{0 . 0 4 3}\end{array}$ & $\begin{array}{l}22.80(15,70,29.91) \\
38.77(31.81,45.72) \\
15.97(9.92,22.01) \\
P<0.001\end{array}$ & $\begin{array}{l}32.22(24.55,39.88) \\
44.99(37.49,52.50) \\
12.78(6.25,19.30) \\
P<0.001\end{array}$ \\
\hline
\end{tabular}




\begin{tabular}{|c|c|c|c|c|c|c|}
\hline $\begin{array}{l}\text { Housing Situation } \\
\text { Owner/Private or Social rent } \\
\text { No fixed abode/other } \\
\text { Mean Difference }(95 \% \mathrm{Cl}) \\
P \text {-value }\end{array}$ & $\begin{array}{l}25.44(22.25,28.62) \\
39.44(28.67,50.20) \\
14.00(3.13,24.86) \\
P=\mathbf{0 . 0 1 2}\end{array}$ & $\begin{array}{l}27.17(22.25,32.09) \\
42.38(25.75,59.01) \\
15.21(-1.58,31.99) \\
P=0.076\end{array}$ & $\begin{array}{l}29.81(25.38,34.23) \\
52.26(37.32,67.21) \\
22.46(7.37,37.54) \\
P=0.004\end{array}$ & $\begin{array}{l}41.78(37.44,46.12) \\
58.85(44.18,73.53) \\
17.07(2.26,31.89) \\
P=0.024\end{array}$ & $\begin{array}{l}24.82(21.32,28.31) \\
36.76(24.95,48.56) \\
11.94(0.02,23.86) \\
P=0.050\end{array}$ & $\begin{array}{l}30.56(26.79,34.33) \\
46.65(33.91,59.39) \\
16.09(3.23,28.95) \\
P=0.014\end{array}$ \\
\hline $\begin{array}{l}\text { Education } \\
\text { Degree level or above } \\
\text { Any other qualification } \\
\text { Mean Difference }(95 \% \mathrm{Cl}) \\
P \text {-value }\end{array}$ & $\begin{array}{l}31.05(25.00,37.09) \\
33.83(27.64,40.01) \\
2.78(-1.18,6.74) \\
P=0.169\end{array}$ & $\begin{array}{l}32.66(23.32,42.01) \\
36.89(27.33,46.44) \\
4.22(-1.90,10.34) \\
P=0.176\end{array}$ & $\begin{array}{l}41.82(33.42,50.21) \\
40.25(31.67,48.84) \\
-1.56(-7.06,3.94) \\
P=0.578\end{array}$ & $\begin{array}{l}51.13(42.89,59.38) \\
49.50(41.07,57.93) \\
-1.63(-7.03,3.77) \\
P=0.554\end{array}$ & $\begin{array}{l}29.82(23.19,36.45) \\
31.75(24.97,38.54) \\
1.93(-2.41,6.28) \\
P=0.384\end{array}$ & $\begin{array}{l}38.48(31.32,45.64) \\
38.73(31.41,46.05) \\
0.25(-4.44,4.95) \\
P=0.917\end{array}$ \\
\hline $\begin{array}{l}\text { Care and Support } \\
\text { Does not receive/provide care } \\
\text { Receives or provides care } \\
\text { Mean Difference }(95 \% \mathrm{Cl}) \\
P \text {-value }\end{array}$ & $\begin{array}{l}31.90(25.66,38.14) \\
32.97(26.54,39.41) \\
1.07(-4.10,6.26) \\
P=0.684\end{array}$ & $\begin{array}{l}34.98(25.34,44.62) \\
34.57(24.63,44.51) \\
-0.41(-8.41,7.58) \\
P=0.919\end{array}$ & $\begin{array}{l}40.46(31.79,49.12) \\
41.61(32.68,50.55) \\
1.16(-6.03,8.35) \\
P=0.752\end{array}$ & $\begin{array}{l}49.03(40.52,57.54) \\
51.60(42.83,60.37) \\
2.56(-4.49,9.62) \\
P=0.476\end{array}$ & $\begin{array}{l}28.76(21.91,35.61) \\
32.81(25.75,39.87) \\
4.05(-1.62,9.73) \\
P=0.162\end{array}$ & $\begin{array}{l}35.74(28.36,43.13) \\
41.47(33.85,49.08) \\
5.72(-0.40,11.85) \\
P=0.067\end{array}$ \\
\hline $\begin{array}{l}\text { Benefits } \\
\text { Does not receive benefits } \\
\text { Receives benefits } \\
\text { Mean Difference }(95 \% \mathrm{Cl}) \\
\text { P-value }\end{array}$ & $\begin{array}{l}28.59(22.05,35.13) \\
36.28(29.87,42.70) \\
7.70(1.88,13.52) \\
P=\mathbf{0 . 0 1 0}\end{array}$ & $\begin{array}{l}28.41(18.31,38.52) \\
41.14(31.23,51.04) \\
12.72(3.73,21.72) \\
P=0.006\end{array}$ & $\begin{array}{l}36.90(27.81,45.98) \\
45.17(36.27,54.08) \\
8.27(0.19,16.36) \\
P=0.045\end{array}$ & $\begin{array}{l}47.05(38.13,55.97) \\
53.58(44.84,62.33) \\
6.53(-1.40,14.47) \\
P=0.107\end{array}$ & $\begin{array}{l}25.49(18.32,32.67) \\
36.08(29.04,43.11) \\
10.58(4.19,16.97) \\
P=0.001\end{array}$ & $\begin{array}{l}34.34(26.60,42.08) \\
42.87(35.28,50.45) \\
8.52(1.63,15.41) \\
P=0.015\end{array}$ \\
\hline $\begin{array}{l}\text { Rehabilitation } \\
\text { No rehab in past year } \\
\text { Received rehab in past year } \\
\text { Mean Difference }(95 \% \mathrm{Cl}) \\
P \text {-value }\end{array}$ & $\begin{array}{l}28.33(22.37,34.28) \\
36.54(30.20,42.89) \\
8.22(4.03,12.40) \\
P<0.001\end{array}$ & $\begin{array}{l}30.30(21.10,39.50) \\
39.25(29.45,49.05) \\
8.95(\mathbf{2 . 4 9 , 1 5 . 4 2 )} \\
P=\mathbf{0 . 0 0 7}\end{array}$ & $\begin{array}{l}38.29(30.03,46.56) \\
43.78(34.97,52.58) \\
5.48(-0.33,11.29) \\
P=0.064\end{array}$ & $\begin{array}{l}46.16(38.04,54.28) \\
54.47(45.82,63.12) \\
8.31(2.60,14.01) \\
P=0.004\end{array}$ & $\begin{array}{l}25.96(19.43,32.49) \\
35.61(28.65,42.57) \\
9.65(5.06,14.24) \\
P<0.001\end{array}$ & $\begin{array}{l}34.23(27.18,41.28) \\
42.98(35.47,50.49) \\
8.75(3.80,13.70) \\
P=0.001\end{array}$ \\
\hline \multicolumn{7}{|c|}{ SD = Standard Deviation } \\
\hline
\end{tabular}




\section{Discussion}

Disability was experienced and reported by people living with HIV accessing routine inperson outpatient HIV care in London UK. Our results are the first known to report that a sample of adults living with well controlled HIV in the UK, of any age, experience disability that is multi-dimensional and episodic in nature, using generic and HIV specific measures of disability. Disability is a universal human experience whereby individuals can be positioned on a continuum of functioning from no disability (full functioning) to complete disability (lack of function), and either currently experience or may be vulnerable to experiencing disability over the course of life [44]. Diagnosis of signs and symptoms are essential, but what most often matters is what a person can, or cannot do, in their daily life [44]. As such, our results might enable researchers, clinicians, policy makers, and people living with HIV, to better understand the nature and extent of disability among people living with HIV in the UK, consider disability-inclusive approaches to HIV care, and promote future research and national census data that includes functioning and disability measurement tools.

Prevalence of severe disability among this sample of people living with HIV was $39.5 \%$. There are no national or international comparisons using the EADD as a measure of disability among people living with HIV. The sample of people living with HIV in this study, who were mostly of working age, economically active, and living in London, had higher estimated disability prevalence than the UK general population measured by the EADD which was estimated to be $22 \%$ [67]. Estimations are known to vary in the UK general population by age group and location; $19 \%$ of working-age adults, $45 \%$ of state pension age adults, and $15 \%$ of people living in London [67]. Limited inferences can be drawn from this data as our study did not include a control group, and no previous disability estimates exist among people living with HIV in the UK. Future research should consider matched HIV-negative control groups, to evaluate group differences, when disability prevalence is measured with EADD. Our study provides important initial estimations of severe disability among people living with HIV accessing in-person outpatient HIV clinics in the UK, for comparison to the UK general population, whereby people who experience severe disability may benefit from rehabilitation $[27,82,83]$. 
Prevalence of moderate disability among people living with HIV was $70.5 \%$. This estimate was higher than disability prevalence similarly measured with $\geq 2$ on WHODAS scores, among people living with HIV on long-term antiretroviral therapy in KwaZulu-Natal and Gauteng province in South Africa, estimated to be $35.5 \%$ and $51.9 \%$ respectively $[27,28]$. Higher estimated prevalence of disability in our study measured by the WHODAS compared to South Africa, may be driven by participants living with HIV in our study reporting greater functional limitations at lower difficulty levels. In our study, 50.5\% reported 4 or more functional limitations with mostly mild (36.8\%) or moderate (33.7\%) difficulty levels. In KwaZulu-Natal, $12.6 \%$ reported 4 or more functional limitations with $64.7 \%$ reporting moderate difficulty level [28]. Estimated prevalence of any level of functional limitation (scoring $\geq 1$ on WHODAS) was $86.6 \%$ and $51.3 \%$ in the United States and South Africa respectively, with people living with HIV in the United States 9.76 times $(95 \% \mathrm{Cl}: 4.91,19.41)$ more likely to experience any level of functional limitation [29]. In our study, 79.5\% reported any level of functional limitation. Caution should be applied when comparing these results from different contexts, employing varied measurement approaches. Our study provides important initial estimations of moderate disability (scoring $\geq 2$ on WHODAS), suggesting that moderate disability is common among people living with HIV accessing routine in-person outpatient HIV care in London UK, and people with moderate disability may benefit from rehabilitation $[27,82,83]$.

High-income settings frequently report higher rates of disability than Low- and Middleincome settings, reportedly due to ageing populations and higher survival rates for people with disabling conditions [38]. Additionally, between country differences can impact disability presence due to differences in perceptions, lived experiences, culture, lifestyle, economies, resources, education, access to and availability of antiretroviral therapy, and health policies [29]. The three disability measurement approaches used in our study provides a breadth of within-country data. This might enable critically comparative future evaluations with other populations across High- Middle- and Low-income settings. For example our study provides the first known WHODAS "simple" and "complex" sum scores among people living with HIV in the UK. The WHODAS "simple" sum score (mean 9.4, SD 11.3) (median 5.0, 25$75^{\text {th }}$ percentile: 1.0-12.0) were higher (meaning more disability) than men and women in the total population (mean 3.1, SD 5.3) comprising any age group including adults aged 75-85 
years (mean 5.7, SD 7.1), people with $\geq 1$ 12-month mental disorder (mean 8.7, SD 7,7), people with any mental disorder (mean 6.3, SD 7.1), people with $\geq 1$ chronic physical condition (mean 5.8, SD 7.0), and people with any physical condition (mean 4.3, SD 6.1) [59]. The WHODAS “complex" sum score (mean 19.6, SD 23.7), (median 10.4, 25-75 ${ }^{\text {th }}$ percentile: 2.1-25.6) were lower (meaning less disability) than people living with HIV in Canada (median $30,25-75^{\text {th }}$ percentile: $18-44$ ) [53], comparable to people living with HIV in Ireland (median $12,25-75^{\text {th }}$ percentile: 5-24) [53], and higher than people living with HIV in KwaZulu-Natal (mean 1.6) and Gauteng province (mean 0.5, SD 8) South Africa [27,28]. There is significant variation in the scoring and reporting of WHODAS sum scores among people living with HIV [27-29,53,84,85], and careful attention should be applied to any comparison. Our approach used a range of measurements to permit collation of existing literature, plus contribute to establishing reproducible standards of approach, scoring, and reporting of disability in the context of HIV.

When measuring severity of disability, the HDQ domain of uncertainty is the most severe dimension of disability experienced in our study of adults living with HIV in London, UK, which is consistent with existing literature from the UK [35], Canada [53,63,79], Ireland [53], and United States [64]. Furthermore, the HDQ domains of challenges to social participation, and mental and emotional health, are within the top three most severe disability dimensions across the same four settings. Uncertainty is a unique dimension of disability within the Episodic Disability Framework [2], whereby people ageing with HIV may worry about HIV specific age-related uncertainties and the trajectory of episodic disability $[86,87]$. Further exploration is warranted into the experiences of uncertainty across the life course among people living with HIV in the UK [35].

To our knowledge our study was the first to examine risk factors of disability across different measures of disability among people living with HIV in the UK. Our results indicated that risk factors of disability can be divided into three different areas; HIV characteristics such as late diagnosis; social determinants of health including unemployment, housing insecurity, and receiving benefits; and personal factors such as age and gender. 
medRxiv preprint doi: https://doi.org/10.1101/2021.09.14.21263587; this version posted September 17, 2021. The copyright holder for this

preprint (which was not certified by peer review) is the author/funder, who has granted medRxiv a license to display the preprint in perpetuity.

It is made available under a CC-BY-ND 4.0 International license .

The HIV characteristic of receiving a late HIV diagnosis resulted in an $171 \%$ increased risk of severe disability. Late HIV diagnosis is associated with negative clinical and societal consequences $[88,89]$, and further associated with disability among older adults $[90,91]$. Our results indicated that the negative consequences of late HIV diagnosis may result in severe disability at any age, including among those who later attain undetectable viral load through antiretroviral therapy. Additionally, people with disabilities are known to be more vulnerable to acquiring HIV, are diverse in their HIV-risks, and more likely to receive a late HIV diagnosis [92-94].

Social determinants of health were risk factors across all measures of disability in our study. Receiving benefits resulted in $187 \%$ increased risk of severe disability, and economic inactivity resulted in $179 \%$ increased risk of severe disability, and $214 \%$ increased risk of moderate disability. Furthermore, economic inactivity, receiving benefits, and having no fixed abode were risk factors across physical, mental and emotional, difficulty with day-today activities, and challenges to social participation HDQ disability domains. Our results align with existing literature highlighting the role and importance of social determinants of health [95], whereby social determinants such as low level of socioeconomic status, stigma, and social exclusion, influence health outcomes and quality of life among people living with HIV [96-98]. Our results emphasise the role of social determents of health influencing disability experienced by people living with HIV in the UK.

Personal factors of identifying as a woman and being aged $<50$ years were both risk factors for HDQ disability domains mental and emotional health, uncertainty, and challenges with social participation. Identifying as a woman was also a risk factor for physical health challenges. Our results are consistent with identifying as a woman predicting disability $[27,28]$ and younger age negatively influencing quality of life [97] among people living with HIV. This is the first known study including trans women, to asociate identifying as a woman and younger age with increased risk of mental and emotional health challenges, uncertainty, and challenges with social participation. Women living with HIV experiencing disability reported more concerns with relationships and household activities [99], physical health challenges were associated with activity limitations [28], and women may lack necessary support networks due to experiences of abandonment from partners or families after 
disclosing HIV or disability status $[99,100]$. Future research is needed to understand the intersectionality of disability and gender among people living with HIV in the UK.

Psychometric evaluation of the HDQ among women living with HIV in the UK is needed [35].

Receiving rehabilitation services in the form of Physiotherapy, Occupational Therapy and Speech and Language Therapy in the past 12-months was found to be a predictor of disability in all models. While our cross-sectional methodology means it is not possible to determine direction of dependence between variables [101], we expect that disability was a likely predictor of participants accessing rehabilitation in this study. Ultimately, the directionality of dependence between variables may be nuanced and influenced by factors including health status, age, gender identity, socio-cultural context, economic environment, and geo-political landscape. This requires multi-stakeholder engagement to include disability within future longitudinal epidemiological HIV research and census data.

Results from the current study need to be interpreted in the context of limitations. Firstly, disability was measured through self-reported measurement tools, which may be susceptible to social desirability, recall inaccuracies and overestimate ability $[30,102,103]$. The use of self-reported measurements however does offer opportunities to ensure patients voices are at the heart of healthcare models that need to adapt to the changing reality, in which people living with HIV will age with viral suppression [104]. Secondly, our study used EADD not Washington Group short set of questions as recommended by the United Nations to estimate disability prevalence [46]. Comparison of these different approaches identifies substantially different, although overlapping, groups as experiencing disability, attributed partly to the exclusion of mental health issues and partly to the higher severity threshold for inclusion in the Washington Group short set. Therefore, the EADD meets Great Britain's need for disability monitoring [37]. Thirdly, our definition of severe disability using EADD and moderate disability by scoring $\geq 2$ on WHODAS [28] was arbitrary without validation. Nevertheless the WHODAS cutoff has been used in other studies and this criterion provides opportunity for global comparison of self-reported disability among people living with HIV. We also included a continuous measure of disability (HDQ) in this study where interpretability of the HDQ scores as they relate to moderate or severe disability are unknown. Fourthly, we used convenience sampling with adults accessing in-person 
outpatient HIV care, therefore the sample may not be representative of people who do not attend in-person or the wider population of people living with HIV in the UK [105].

Nevertheless, our study provides initial estimations of disability prevalence enabling future requisite sample size calculations. Fifthly, with our cross-sectional methodology and no HIVnegative control group, no causal inferences could be established. Future research should consider recruiting a matched HIV-negative control group to evaluate difference. Lastly, this study was positioned within the theoretical framework of the ICF, which has been argued to perpetuate notions of normal/abnormal through the application of simplistic views of disability, thus reinforcing the pervasive belief that disabled bodies are inherently problematic and in need of intervention [106]. Our results do not intend to be oppressive, compound the double burden of stigma and discrimination associated with both HIV and disability [107], or focus on approaches to normalise disabled bodies [108]. Rather we aimed to provide a lens by which the self-reported functioning and disability experienced by people living with HIV in the UK can enhance person-centred HIV care, encourage disability assessment during routine HIV care, and address unmet need.

\section{Conclusion}

People living with well-controlled HIV of any age, attending outpatient HIV care in London UK, experience and self-report multi-dimensional and episodic disability. Initial prevalence estimates of severe (39.5\%) and moderate disability (70.5\%), suggested disability is commonly experienced among adults accessing routine HIV care, with uncertainty the most severe domain of disability experienced. Risk factors of disability included gender, age, employment, housing, benefits, and late HIV diagnosis among people living with HIV in the UK. Results help to better understand the prevalence, severity, and risk factors of disability experienced among adults living with HIV, and identify areas in which to target interventions to reduce disability and improve optimal health and function beyond viral suppression for adults living with HIV. 


\section{References}

1. Deeks SG, Lewin SR, Havlir DV. The end of AIDS: HIV infection as a chronic disease. The Lancet. 2013;382: 1525-1533.

2. O'Brien KK, Bayoumi AM, Strike C, Young NL, Davis AM. Exploring disability from the perspective of adults living with HIV/AIDS: development of a conceptual framework. Health and Quality of Life Outcomes. 2008;6: 76.

3. O'Brien KK, Davis AM, Strike C, Young NL, Bayoumi AM. Putting episodic disability into context: a qualitative study exploring factors that influence disability experienced by adults living with HIV/AIDS. J Int AIDS Soc. 2009;12: 5-2652. doi: 10.1186/1758-2652-2-5 [doi].

4. UNAIDS. Global HIV \& AIDS statistics - 2019 fact sheet. . 2019. Available:

https://www.unaids.org/en/resources/fact-sheet.

5. Trickey A, May MT, Vehreschild J, Obel N, Gill MJ, Crane HM, et al. Survival of HIV-positive patients starting antiretroviral therapy between 1996 and 2013: a collaborative analysis of cohort studies. The Lancet HIV. 2017;4: e349-e356.

6. Thurn M, Gustafson DR. Faces of frailty in aging with HIV infection. Current HIV/AIDS Reports. 2017;14: 31-37.

7. Autenrieth CS, Beck EJ, Stelzle D, Mallouris C, Mahy M, Ghys P. Global and regional trends of people living with HIV aged 50 and over: Estimates and projections for 2000-2020. PloS one. 2018;13: e0207005.

8. Yin Z, Kall M, Skingsley A, Dalpech V. Over half of people in HIV care in the United Kingdom by 2028 will be aged 50 years or above. . 2015. Available:

http://www.ucl.ac.uk/voices/pdfs/HIV care.

9. Smit M, Brinkman K, Geerlings S, Smit C, Thyagarajan K, van Sighem A, et al. Future challenges for clinical care of an ageing population infected with HIV: a modelling study. The Lancet Infectious Diseases. 2015;15: 810-818.

10. Nixon SA, Hanass-Hancock J, Whiteside A, Barnett T. The increasing chronicity of HIV in sub-Saharan Africa: Re-thinking" HIV as a long-wave event" in the era of widespread access to ART. Globalization and health. 2011;7: 41.

11. Guaraldi G, Malagoli A, Calcagno A, Mussi C, Celesia B, Carli F, et al. The increasing burden and complexity of multi-morbidity and polypharmacy in geriatric HIV patients: a cross sectional study of people aged $65 \hat{a} €$ "74 years and more than 75 years. BMC geriatrics. 2018;18: 99.

12. Shah ASV, Stelzle D, Lee KK, Beck EJ, Alam S, Clifford S, et al. Global Burden of Atherosclerotic Cardiovascular Disease in People Living with the Human Immunodeficiency 
medRxiv preprint doi: https://doi.org/10.1101/2021.09.14.21263587; this version posted September 17, 2021. The copyright holder for this preprint (which was not certified by peer review) is the author/funder, who has granted medRxiv a license to display the preprint in perpetuity.

It is made available under a CC-BY-ND 4.0 International license .

Virus: A Systematic Review and Meta-Analysis. Circulation. 2018. doi: CIRCULATIONAHA.117.033369.

13. Duncan AD, Goff LM, Peters BS. Type 2 diabetes prevalence and its risk factors in HIV: A cross-sectional study. PLoS One. 2018;13: e0194199. doi: 10.1371/journal.pone.0194199.

14. Mallon PW. Aging with HIV: osteoporosis and fractures. Curr Opin HIV AIDS. 2014;9: 428435. doi: $10.1097 / \mathrm{COH} .0000000000000080$.

15. Compston J. HIV infection and bone disease. J Intern Med. 2016;280: 350-358.

16. Nightingale S, Winston A, Letendre S, Michael BD, McArthur JC, Khoo S, et al. Controversies in HIV-associated neurocognitive disorders. The Lancet Neurology. 2014;13: 1139-1151.

17. Saylor D, Dickens AM, Sacktor N, Haughey N, Slusher B, Pletnikov M, et al. HIV-associated neurocognitive disorder, pathogenesis and prospects for treatment. Nature Reviews Neurology. 2016;12: 234-248.

18. Sabin CA, Harding R, Bagkeris E, Nkhoma K, Post FA, Sachikonye M, et al. Pain in people living with HIV and its association with healthcare resource use, well being and functional status. AIDS. 2018;32: 2697-2706. doi: 10.1097/QAD.0000000000002021.

19. Chuah FLH, Haldane VE, Cervero-Liceras F, Ong SE, Sigfrid LA, Murphy G, et al. Interventions and approaches to integrating HIV and mental health services: a systematic review. Health Policy Plan. 2017.

20. Silverberg MJ, Lau B, Achenbach CJ, Jing Y, Althoff KN, D'souza G, et al. Cumulative Incidence of Cancer Among Persons With HIV in North AmericaA Cohort StudyCumulative Incidence of Cancer Among Persons With HIV in North America. Ann Intern Med. 2015;163: 507-518.

21. Bloch M. Frailty in people living with HIV. AIDS research and therapy. 2018;15: 1-5.

22. Shippy RA, Karpiak SE. The aging HIV/AIDS population: fragile social networks. Aging \& mental health. 2010.

23. Roger KS, Mignone J, Kirkland S. Social aspects of HIV/AIDS and aging: a thematic review. Canadian Journal on Aging/La Revue canadienne du vieillissement. 2013;32: 298-306.

24. Siemon JS, Blenkhorn L, Wilkins S, O'Brien KK, Solomon PE. A grounded theory of social participation among older women living with HIV/Une théorie ancrée pour analyser la participation sociale chez les femmes âgées atteintes du VIH. Canadian Journal of Occupational Therapy. 2013;80: 241-250.

25. Emlet CA. "You're awfully old to have this disease": Experiences of stigma and ageism in adults 50 years and older living with HIV/AIDS. Gerontologist. 2006;46: 781-790. 
medRxiv preprint doi: https://doi.org/10.1101/2021.09.14.21263587; this version posted September 17, 2021. The copyright holder for this preprint (which was not certified by peer review) is the author/funder, who has granted medRxiv a license to display the preprint in perpetuity.

It is made available under a CC-BY-ND 4.0 International license .

26. Banks LM, Zuurmond M, Ferrand R, Kuper H. Knowledge of HIV-related disabilities and challenges in accessing care: Qualitative research from Zimbabwe. PloS one. 2017;12: e0181144.

27. Myezwa H, Hanass-Hancock J, Ajidahun AT, Carpenter B. Disability and health outcomesfrom a cohort of people on long-term antiretroviral therapy. SAHARA-J: Journal of Social Aspects of HIV/AIDS. 2018;15: 50-59.

28. Hanass-Hancock J, Myezwa H, Carpenter B. Disability and living with HIV: baseline from a cohort of people on long term ART in South Africa. PloS one. 2015;10: e0143936.

29. Kietrys D, Myezwa H, Galantino ML, Parrott JS, Davis T, Levin T, et al. Functional Limitations and Disability in Persons Living with HIV in South Africa and United States: Similarities and Differences. Journal of the International Association of Providers of AIDS Care (JIAPAC). 2019;18: 2325958219850558.

30. Mactaggart I, Kuper H, Murthy G, Oye J, Polack S. Measuring disability in population based surveys: the interrelationship between clinical impairments and reported functional limitations in Cameroon and India. PloS one. 2016;11: e0164470.

31. World Health Organization. How to use the ICF: a practical manual for using the International Classification of Functioning, Disability and Health (ICF). Exposure draft for comment. October 2013. Geneva: WHO. . 2013. Available:

http://www.who.int/classifications/drafticfpracticalmanual.pdf.

32. Palmer M, Harley D. Models and measurement in disability: an international review. Health Policy Plan. 2011;27: 357-364.

33. Oliver M. Understanding disability: From theory to practice: Macmillan International Higher Education; 1995.

34. World Health Organization. Towards a common language for functioning, disability, and health: ICF. The international classification of functioning, disability and health. 2002.

35. Brown DA, Simmons B, Boffito M, Aubry R, Nwokolo N, Harding R, et al. Evaluation of the psychometric properties of the HIV Disability Questionnaire among adults living with HIV in the United Kingdom: A cross-sectional self-report measurement study. PloS one. 2019;14: e0213222.

36. Joint United Nations Programme on HIV/AIDS (UNAIDS). Disability and HIV. Geneva: UNAIDS. 2017.

37. Office for National Statistics. Measuring disability: comparing approaches. Comparison of the Equality Act measure of disability used in Great Britain to the United Nations

recommended measures of disability, based on the Opinions and Lifestyle Survey in January 2019. Available:

https://www.ons.gov.uk/peoplepopulationandcommunity/healthandsocialcare/disability/art icles/measuringdisabilitycomparingapproaches/2019-08-06. 
medRxiv preprint doi: https://doi.org/10.1101/2021.09.14.21263587; this version posted September 17, 2021. The copyright holder for this preprint (which was not certified by peer review) is the author/funder, who has granted medRxiv a license to display the preprint in perpetuity.

It is made available under a CC-BY-ND 4.0 International license .

38. Mont D. Measuring disability prevalence (English). SP discussion paper; no. 706. Washington, DC: World Bank. 2007. Available: http://documents.worldbank.org/curated/en/578731468323969519/Measuring-disabilityprevalence.

39. World Health Organization. World report on disability 2011: World Health Organization; 2011.

40. Banks LM, Zuurmond M, Ferrand R, Kuper H. The relationship between HIV and prevalence of disabilities in sub - Saharan Africa: systematic review (FA). Tropical medicine \& international health. 2015;20: 411-429.

41. Madans JH, Loeb ME, Altman BM. Measuring disability and monitoring the UN Convention on the Rights of Persons with Disabilities: the work of the Washington Group on Disability Statistics. . 2011;11: S4.

42. Government Statistical Service. Measuring disability for the Equality Act 2010. . Available: https://gss.civilservice.gov.uk/policy-store/measuring-disability-for-the-equality-act2010/\#questions.

43. Üstün TB, Kostanjsek N, Chatterji S, Rehm J. Measuring health and disability: Manual for WHO disability assessment schedule WHODAS 2.0: World Health Organization; 2010.

44. Cieza A, Sabariego C, Bickenbach J, Chatterji S. Rethinking disability. BMC medicine. 2018;16: 14.

45. O'Brien KK, Bayoumi AM, King K, Alexander R, Solomon P. Community engagement in health status instrument development: experience with the HIV disability questionnaire. Progress in community health partnerships: research, education, and action. 2014;8: 549559.

46. Washington Group on Disability Statistics. Washington Group questions and the Sustainable Develeopment Goals. Dr Daneil Mont. 2017. Available:

http://www.washingtongroup-disability.com/washington-group-blog/sdgs/.

47. UK Government. Definition of disability under the Equality Act 2010. Available: https://www.gov.uk/definition-of-disability-under-equality-act-2010.

48. Kietrys DM, Parrott JS, Galantino ML, Davis T, Levin T, O’Brien KK. Self-Reported Disability in Persons With HIV-Related Neuropathy Is Mediated by Pain Interference and Depression. Phys Ther. 2020.

49. PLOS Medicine Editors LAeng. Observational studies: getting clear about transparency. PLoS Med. 2014;11: e1001711. doi: 10.1371/journal.pmed.1001711.

50. Public Health England. Trends in HIV testing, new diagnoses and people receiving HIVrelated care in the United Kingdom: data to the end of December 2019. 2020. Available: 
medRxiv preprint doi: https://doi.org/10.1101/2021.09.14.21263587; this version posted September 17, 2021. The copyright holder for this preprint (which was not certified by peer review) is the author/funder, who has granted medRxiv a license to display the preprint in perpetuity.

It is made available under a CC-BY-ND 4.0 International license .

https://assets.publishing.service.gov.uk/government/uploads/system/uploads/attachment data/file/931964/hpr2020 hiv19.pdf.

51. Paton M, Lane R. Clinimetrics: World Health Organization Disability Assessment Schedule 2.0. J Physiother. 2020;66: 199. doi: S1836-9553(20)30019-9.

52. Rusch M, Nixon S, Schilder A, Braitstein P, Chan K, Hogg RS. Impairments, activity limitations and participation restrictions: prevalence and associations among persons living with HIV/AIDS in British Columbia. Health and quality of life outcomes. 2004;2: 46.

53. O'Brien KK, Solomon P, Bergin C, O'Dea S, Stratford P, Iku N, et al. Reliability and validity of a new HIV-specific questionnaire with adults living with HIV in Canada and Ireland: the HIV Disability Questionnaire (HDQ). Health and quality of life outcomes. 2015;13: 124.

54. Cobbing S, Hanass-Hancock J, Myezwa H. A Home-Based Rehabilitation Intervention for Adults Living With HIV: A Randomized Controlled Trial. J Assoc Nurses AIDS Care. 2017;28: 105-117. doi: 10.1016/j.jana.2016.08.008.

55. Hanass-Hancock J, Misselhorn A, Carpenter B, Myezwa H. Determinants of livelihood in the era of widespread access to ART. AIDS Care. 2017;29: 32-39.

56. Peltzer K, Phaswana-Mafuya N. Levels of ability and functioning of persons living with HIV and AIDS using the WHO DAS II in a South African context. Journal of social development in Africa. 2008;23.

57. Barbosa KSS, Castro SS, Leite CF, Nacci FR, Accioly MF. Validation of the Brazilian version of the World Health Organization Disability Assessment Schedule 2.0 for individuals with HIV/AIDS. Cien Saude Colet. 2020;25: 837-844. doi: S1413-81232020000300837.

58. Carpenter BS, Hanass-Hancock J, Myezwa H. Looking at antiretroviral adherence through a disability lens: a cross-sectional analysis of the intersection of disability, adherence, and health status. Disabil Rehabil. 2020;42: 806-813.

59. Andrews G, Kemp A, Sunderland M, Von Korff M, Ustun TB. Normative data for the 12 item WHO Disability Assessment Schedule 2.0. PloS one. 2009;4: e8343.

60. Federici S, Bracalenti M, Meloni F, Luciano JV. World Health Organization disability assessment schedule 2.0: An international systematic review. Disabil Rehabil, 2017 Nov;39(23):2347-2380. doi: 10.1080/09638288.2016.1223177. Epub 2016 Nov 7.

61. Garin O, Ayuso-Mateos JL, Almansa J, Nieto M, Chatterji S, Vilagut G, et al. Validation of the" World Health Organization Disability Assessment Schedule, WHODAS-2" in patients with chronic diseases. Health and Quality of Life Outcomes. 2010;8: 51.

62. O'Brien KK, Solomon P, Bayoumi AM. Measuring disability experienced by adults living with HIV: assessing construct validity of the HIV Disability Questionnaire using confirmatory factor analysis. BMJ Open. 2014;4: e005456-2014. doi: 10.1136/bmjopen-2014-005456. 
medRxiv preprint doi: https://doi.org/10.1101/2021.09.14.21263587; this version posted September 17, 2021. The copyright holder for this preprint (which was not certified by peer review) is the author/funder, who has granted medRxiv a license to display the preprint in perpetuity.

It is made available under a CC-BY-ND 4.0 International license .

63. O’Brien KK, Bayoumi AM, Bereket T, Swinton M, Alexander R, King K, et al. Sensibility assessment of the HIV Disability Questionnaire. Disabil Rehabil. 2013;35: 566-577.

64. O’Brien KK, Kietrys D, Galantino ML, Parrott JS, Davis T, Tran Q, et al. Reliability and validity of the HIV disability questionnaire (HDQ) with adults living with HIV in the United States. Journal of the International Association of Providers of AIDS Care (JIAPAC). 2019;18: 2325958219888461.

65. Gaskin CJ, Lambert SD, Bowe SJ, Orellana L. Why sample selection matters in exploratory factor analysis: implications for the 12-item World Health Organization Disability Assessment Schedule 2.0. BMC medical research methodology. 2017;17: 40.

66. Axelsson E, Lindsater E, Ljotsson B, Andersson E, Hedman-Lagerl of E. The 12-item SelfReport World Health Organization Disability Assessment Schedule (WHODAS) 2.0 Administered Via the Internet to Individuals With Anxiety and Stress Disorders: A Psychometric Investigation Based on Data From Two Clinical Trials. JMIR Ment Health. 2017;4: e58. doi: 10.2196/mental.7497 [doi].

67. Department for Work and Pensions. Family Resources Survey: financial year 2018/19. . 2020. Available: https://www.gov.uk/government/statistics/family-resources-surveyfinancial-year-201819.

68. Greene M, Covinsky KE, Valcour V, Miao Y, Madamba J, Lampiris H, et al. Geriatric Syndromes in Older HIV-Infected Adults. J Acquir Immune Defic Syndr. 2015;69: 161-167. doi: 10.1097/QAI.0000000000000556.

69. The Lancet H. Preparing for an ageing HIV epidemic. Lancet HIV. 2017;4: e2773018(17)30114. doi: S2352-3018(17)30114-5.

70. BHIVA. British HIV Association (BHIVA) standards of care for people living with HIV. 2018. Available: http://www.bhiva.org/standards-of-care-2018.aspx.

71. Auzenbergs M, Delpech V, Gold D, Kall M, Petretti S, Smithson K, et al. Positive Voices, the national survey of people living with HIV; Changing Perceptions: "Talking about HIV and our needs". 2018. Available: https://img1.wsimg.com/blobby/go/db9f6878-0f44-42a6-b528581039772168/downloads/1csbtcptn 723688.pdf.

72. Government Statistical Service. Sexual Orientation. 2017. Available: https://gss.civilservice.gov.uk/policy-store/sexual-orientation/.

73. Government Statistical Service. Ethnicity. 2017. Available: https://gss.civilservice.gov.uk/guidances/harmonisation/0-harmonised-principles/ethnicity/.

74. Government Statistical Service. Economic activity status, National Statistics Socioeconomic Classification (NS-SEC) and employment related questions. 2018. Available: https://gss.civilservice.gov.uk/guidances/harmonisation/0-harmonised-principles/economicactivity-status-ns-sec-and-employment-related-questions/. 
medRxiv preprint doi: https://doi.org/10.1101/2021.09.14.21263587; this version posted September 17, 2021. The copyright holder for this preprint (which was not certified by peer review) is the author/funder, who has granted medRxiv a license to display the preprint in perpetuity.

It is made available under a CC-BY-ND 4.0 International license .

75. Government Statistical Service. Household accommodation and vehicle information.

2018. Available: https://gss.civilservice.gov.uk/guidances/harmonisation/0-harmonisedprinciples/accommodation-and-household-information/.

76. Government Statistical Service. Education attainment and qualifications. 2018. Available: https://gss.civilservice.gov.uk/guidances/harmonisation/0-harmonised-principles/educationattainment-and-qualifications/.

77. Government Statistical Service. Unpaid Care. 2017. Available:

https://gss.civilservice.gov.uk/guidances/harmonisation/0-harmonised-principles/carers/.

78. Government Statistical Service. Benefits and tax credits. 2018. Available:

https://gss.civilservice.gov.uk/policy-store/benefits-and-tax-credits/.

79. O’Brien KK, Hanna S, Solomon P, Worthington C, Ibáñez-Carrasco F, Carusone SC, et al. Characterizing the disability experience among adults living with HIV: a structural equation model using the HIV disability questionnaire (HDQ) within the HIV, health and rehabilitation survey. BMC infectious diseases. 2019;19: 594.

80. Sedgwick P. Multiple significance tests: the Bonferroni correction. BMJ: British Medical Journal (Online). 2012;344.

81. IBM Corp. IBM SPSS Statistics for Windows, Version 25.0. Armonk, NY: IBM Corp. 2017.

82. Wade DT. What is rehabilitation? An empirical investigation leading to an evidence-based description. Clinical Rehabilitation. 2020;34: 571.

83. Worthington C, Myers T, O'Brien K, Nixon S, Cockerill R. Rehabilitation in HIV/AIDS: development of an expanded conceptual framework. AIDS Patient Care STDS. Invalid date;19: 258-271.

84. Nyirenda $M$, Chatterji S, Falkingham J, Mutevedzi P, Hosegood V, Evandrou M, et al. An investigation of factors associated with the health and well-being of HIV-infected or HIVaffected older people in rural South Africa. BMC Public Health. 2012;12: 259.

85. Mugisha JO, Schatz EJ, Randell M, Kuteesa M, Kowal P, Negin J, et al. Chronic disease, risk factors and disability in adults aged 50 and above living with and without HIV: findings from the Wellbeing of Older People Study in Uganda. Global health action. 2016;9: 31098.

86. Solomon P, O'Brien K, Wilkins S, Gervais N. Aging with HIV and disability: the role of uncertainty. AIDS Care. 2014;26: 240-245.

87. Solomon P, O'Brien KK, Nixon S, Letts L, Baxter L, Gervais N. Trajectories of Episodic Disability in People Aging with HIV: A Longitudinal Qualitative Study. Journal of the International Association of Providers of AIDS Care (JIAPAC). 2018;17: 2325958218759210. 
88. Girardi E, Sabin CA, Monforte AD. Late diagnosis of HIV infection: epidemiological features, consequences and strategies to encourage earlier testing. J Acquir Immune Defic Syndr. 2007;46 Suppl 1: S3-8. doi: 10.1097/01.qai.0000286597.57066.2b.

89. Moreno S, Mocroft A, Monforte A. Medical and societal consequences of late presentation. Antivir Ther (Lond). 2010;15: 9.

90. Erlandson KM, Schrack JA, Jankowski CM, Brown TT, Campbell TB. Functional impairment, disability, and frailty in adults aging with HIV-infection. Curr HIV/AIDS Rep. Invalid date;11: 279-290. doi: 10.1007/s11904-014-0215-y.

91. Avila-Funes JA, Belaunzarán-Zamudio PF, Tamez-Rivera O, Crabtree-Ramírez B, Navarrete-Reyes AP, Cuellar-Rodríguez J, et al. Correlates of prevalent disability among HIVinfected elderly patients. AIDS Res Hum Retroviruses. 2016;32: 155-162.

92. Hanass-Hancock J. Understanding the vulnerability of people with disabilities to HIV: Who is at risk? EClinicalMedicine. 2020;25.

93. De Beaudrap P, Beninguisse G, Mouté C, Temgoua CD, Kayiro PC, Nizigiyimana V, et al. The Multidimensional Vulnerability of Women with Disability to HIV Infection: Results from the HandiSSR Study in Burundi. Available at SSRN 3532543. 2020.

94. Tun W, Okal J, Schenk K, Esantsi S, Mutale F, Kyeremaa RK, et al. Limited accessibility to HIV services for persons with disabilities living with HIV in Ghana, Uganda and Zambia. Journal of the International AIDS Society. 2016;19: 20829.

95. Lazarus JV, Safreed-Harmon K, Kamarulzaman A, Anderson J, Leite RB, Behrens G, et al. Consensus statement on the role of health systems in advancing the long-term well-being of people living with HIV. Nature communications. 2021;12: 1-14.

96. Shokoohi M, Bauer GR, Kaida A, Lacombe-Duncan A, Kazemi M, Gagnier B, et al. Social determinants of health and self-rated health status: A comparison between women with HIV and women without HIV from the general population in Canada. PloS one. 2019;14:

e0213901.

97. Ghiasvand H, Higgs P, Noroozi M, Ghaedamini Harouni G, Hemmat M, Ahounbar E, et al. Social and demographical determinants of quality of life in people who live with HIV/AIDS infection: evidence from a meta-analysis. Biodemography and social biology. 2020;65: 57-72.

98. Abbott LS, Williams CL. Influences of social determinants of health on African Americans living with HIV in the rural southeast: a qualitative meta-synthesis. Journal of the Association of Nurses in AIDS Care. 2015;26: 340-356.

99. Hanass-Hancock J, Myezwa H, Nixon SA, Gibbs A. "When I was no longer able to see and walk, that is when I was affected most": experiences of disability in people living with HIV in South Africa. Disabil Rehabil. 2015;37: 2051-2060. 
100. Yoshida K, Hanass-Hancock J, Nixon S, Bond V. Using intersectionality to explore experiences of disability and HIV among women and men in Zambia. Disabil Rehabil. 2014;36: 2161-2168.

101. Wiedermann W, Merkle EC, von Eye A. Direction of dependence in measurement error models. Br J Math Stat Psychol. 2018;71: 117-145.

102. Thames AD, Becker BW, Marcotte TD, Hines LJ, Foley JM, Ramezani A, et al. Depression, cognition, and self-appraisal of functional abilities in HIV: An examination of subjective appraisal versus objective performance. Clin Neuropsychol. 2011;25: 224-243.

103. Akashi-Ronquest N, Carrillo P, Dembling B, Stern S. Measuring the biases in selfreported disability status: evidence from aggregate data. Applied Economics Letters. 2011;18: 1053-1060.

104. Kall M, Marcellin F, Harding R, Lazarus JV, Carrieri P. Patient-reported outcomes to enhance person-centred HIV care. The Lancet HIV. 2019.

105. Sedgwick P. Convenience sampling. BMJ. 2013;347: f6304.

106. Mosleh D. Critical Disability Studies With Rehabilitation: Re-thinking the human in rehabilitation research and practice.

107. UNAIDS. The GAP Report. 2014. Available:

https://www.unaids.org/sites/default/files/media asset/UNAIDS Gap report en.pdf.

108. Swain J, French S, Barnes C, Thomas C. Disabling barriers-enabling environments: Sage; 2013 